Check for updates

Cite this: Chem. Commun., 2021, 57, 4926

Received 15th February 2021 Accepted 13th April 2021

DOI: $10.1039 / \mathrm{d} 1 \mathrm{cc} 00859 \mathrm{e}$

rsc.li/chemcomm

\section{Autonomous optimisation of a nanoparticle catalysed reduction reaction in continuous flow $\dagger$}

\author{
Brendan L. Hall, (D) Connor J. Taylor, (D) Ricardo Labes, (D) Alexander F. Massey, (D) \\ Robert Menzel, (D) Richard A. Bourne (D) and Thomas W. Chamberlain (D)*
}

\begin{abstract}
An automated continuous flow reactor system equipped with inline analysis, was developed and applied in the self-optimisation of a nanoparticle catalysed reaction. The system was used to optimise the experimental conditions of a gold nanoparticle catalysed 4-nitrophenol reduction reaction, towards maximum conversion in under 2.5 hours. The data obtained from this optimisation was then used to generate a kinetic model, allowing us to predict the outcome of the reaction under different conditions. By combining continuous flow nanoparticle synthesis with this approach, the development timeline for these emerging catalysts could be significantly accelerated.
\end{abstract}

Due to their high energy surfaces and extremely high surface atom to volume ratios, ${ }^{1}$ nanoparticles have been demonstrated as excellent catalysts in a wide variety of chemical transformations, such as: photocatalytic water splitting, ${ }^{2}$ hydrogenations, ${ }^{3}$ oxidations ${ }^{4}$ and cross-coupling reactions. ${ }^{5}$ Over the past two decades there has been an increasing focus on further optimising the performance of catalytic nanoparticles. ${ }^{6}$ These reports often investigate the effects of nanoparticle properties such as size, shape or composition on their performance as catalysts. ${ }^{7-9}$

Nanoparticle catalysed reaction optimisation can be a challenging task. Unlike homogeneous organic or metal-complex based catalysts, nanoparticle catalysts typically present as a non-discrete distribution of structures. These structures can be almost infinitely modified, providing additional variables to explore. The outcome of these reactions depends on both the intrinsic properties of the nanoparticles and the experimental conditions under which the reactions are performed. ${ }^{10}$ Therefore, it is important to compare each discrete catalyst system under a range of different reaction conditions. The difficulty of implementing such an approach arises from the sheer number

Institute for Process Research and Development, School of Chemistry, University of Leeds, Leeds, LS2 9JT, UK. E-mail:T.W.Chamberlain@Leeds.ac.uk; Tel: +44 (0)113 3436468

$\dagger$ Electronic supplementary information (ESI) available: Experimental methodology, automation code, XPS and TEM characterisation. See DOI: 10.1039/ d1cc00859e of experiments required to thoroughly explore the reaction conditions in this way.

Recently, chemists have adopted a range of enabling technologies to assist with repetitive tasks such as reaction analysis and screening. This has led to the development of systems that utilise algorithms for the automated optimisation of reactions, particularly in the area of continuous flow research. ${ }^{11}$ Automated continuous flow systems have been developed for the self-optimisation of a wide variety of reactions ${ }^{12,13}$ and have led to accelerated chemical process development timelines. ${ }^{14}$

One of the first reported uses of a self-optimising continuous flow system was for the synthesis of cadmium-selenium quantum dot nanoparticles. Where a quantum dot synthesis method was optimised to produce nanoparticles with a maximum fluorescence intensity for a selected emission wavelength. ${ }^{15}$ Continuous flow reactors more generally, have become popular platforms for the synthesis of materials and nanoparticles in particular owing to their superior heat and mass transfer properties. ${ }^{16-19}$ Examples include the synthesis of metal, ${ }^{20}$ bimetallic, ${ }^{21}$ quantum $\operatorname{dot}^{22}$ and metal oxide ${ }^{23}$ nanoparticles.

In this communication we report what is, to the best of our knowledge, the first example of a self-optimising continuous flow reactor for the optimisation of a nanoparticle catalysed reaction. The general workflow of this approach begins as a window of operating conditions is defined for the optimisation. Automated HPLC pumps then pump nanoparticles and reagents into a tubular reactor. The reactor outlet stream is analysed with an inline analytical technique, in this case UV-vis. The resulting analysis data is then interpreted to determine, for example, conversion or selectivity and the results are fed to an optimisation algorithm that determines the next array of experimental conditions to be set by the system. This automated process is repeated iteratively until an optimum solution is obtained.

To demonstrate our system, the conditions of a gold nanoparticle (AuNP) catalysed 4-nitrophenol (4-NP) reduction reaction were optimised for maximum conversion. Reduction reactions are an important class of reactions with special relevance in 


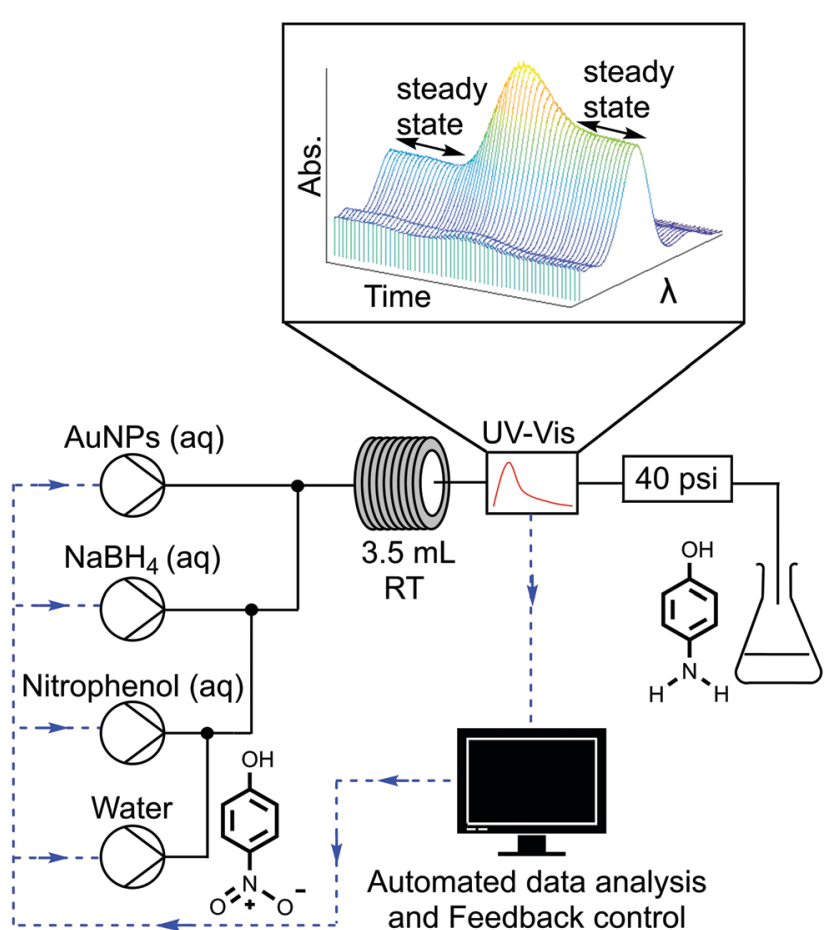

Fig. 1 A schematic describing the self-optimising system. The experimental conditions are changed by altering the flow rates of the automated pumps, the reaction is monitored with an inline UV-vis spectrometer and optimised by closed-loop feedback control, based on the reaction conversion.

synthetic drug development. ${ }^{24}$ 4-NP reductions are commonly used as a benchmark reaction for comparing the catalytic performance of different nanoparticle catalysts. ${ }^{25}$ The AuNPs in this study were synthesised using a Turkevich protocol ${ }^{26}$ and confirmed by TEM to have an average diameter of $15.7 \pm 5.5 \mathrm{~nm}$ (ESI, $\dagger$ Section 2.4).

The closed loop optimisation system is shown in Fig. 1. Automated pumps were used to flow aqueous solutions of the AuNPs, reducing agent $\mathrm{NaBH}_{4}$ and 4-NP into a tubular flow reactor (PTFE, $0.8 \mathrm{~mm} \mathrm{ID,} 3.5 \mathrm{~mL}$ volume). Altering the pump flow rates allowed different concentrations of reagents as well as different residence times to be explored. The active surface area of the nanoparticles present in the reaction was correlated to the concentration of Au present using a geometric approximation (ESI, $\dagger$ Section 2.2), based on the shape and average nanoparticle size determined by TEM analysis. The AuNPs were characterised with XPS, revealing the surface AuNP atoms to be present in the $\mathrm{Au}(0)$ oxidation state, both before and after the reaction (ESI, $\dagger$ Section 2.5). Further TEM characterization was performed to confirm there was no change in nanoparticle size after the nanoparticles were used to catalyse the reaction (ESI, $\uparrow$ Section 2.4). A water dilution pump was also included to increase the range of conditions that could be attained by the system, as not all combinations of factors could be achieved without a diluent i.e. particular concentrations and residence times. The global optimisation algorithm Stable Noisy Optimisation by Branch and Fit (SNOBFIT) ${ }^{27}$ was used to optimise the reaction, allowing us to demonstrate the platforms ability to
Table 1 The lower and upper variable bounds set for the optimisation in this study

\begin{tabular}{llll}
\hline & $\begin{array}{l}\text { AuNP SA } \\
\left(\mathrm{m}^{2} \mathrm{~L}^{-1}\right)\end{array}$ & $\begin{array}{l}\mathrm{NaBH}_{4} \text { conc. } \\
(\mathrm{mM})\end{array}$ & $\begin{array}{l}\text { Res. time } \\
(\mathrm{min})\end{array}$ \\
\hline Upper bound & 0.16 & 2.5 & 3 \\
Lower bound & 0.04 & 0.5 & 1
\end{tabular}

explore a wide range of reaction variables within the design space shown in Table 1.

The $\mathrm{NaBH}_{4}$ solution was kept in an ice bath and adjusted to $\mathrm{pH} 10$ with a solution of sodium hydroxide $(0.1 \mathrm{M})$, to prevent hydrolysis when stored in the reactor reservoir. The initial concentration of 4-NP $(0.06 \mathrm{mM})$ was kept the same for each experiment to simplify analysis. The outlet of the reactor was monitored with an inline UV-vis absorption spectrometer, this allowed real time monitoring of 4-NP concentration and verification of steady state conditions.

Reaction conversion was determined by integrating the UV-vis absorption profile of 4-NP between $350-450 \mathrm{~nm}$. As the AuNP and 4-NP absorption bands overlap within this range, the measured UV-vis spectra were deconvoluted by subtracting an AuNP reference band that was scaled to match the AuNP peak intensity in the measured spectrum between $500-560 \mathrm{~nm}$ (ESI, $\dagger$ Section 5).

The optimisation was performed in under 2.5 hours and the outcome of the individual experiments are displayed in Fig. 2. A trend towards the highest conversion (95\%) was found at longer residence times, with higher AuNP surface areas and $\mathrm{NaBH}_{4}$ concentrations. These results fit well with the findings of previously reported studies, ${ }^{28,29}$ demonstrating clearly the systems capability for nanoparticle catalysed reaction optimisation.

The global optimisation algorithm used in this study explored a wide range of conditions within the experimental design space. Therefore, it was possible to use this data set to identify kinetic parameters and obtain a high degree of understanding of the reaction system. Previous studies have shown

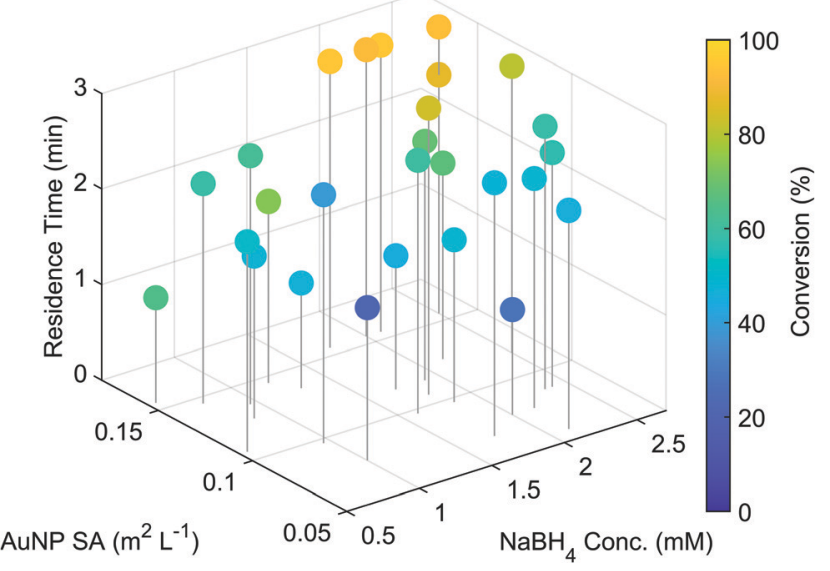

Fig. 2 A plot showing the outcome of the self-optimisation. Each coloured point represents an individual experiment performed by the system. A trend towards increasing reaction conversion was observed at longer residence times with greater concentrations of reducing agent and higher AuNP surface areas (SA). 


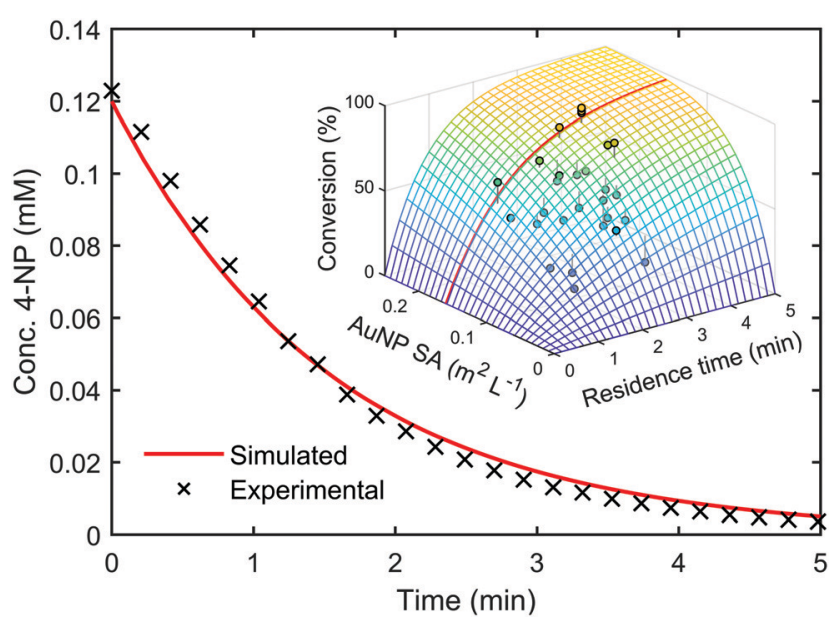

Fig. 3 The outer line plot shows the experimental vs. simulated reaction data for a AuNP catalysed nitrophenol reduction reaction performed in batch. The inner mesh plot shows the predicted effect of changing the AuNP surface area (SA) in the system and residence time on the reaction conversion, overlaid with data from the optimisation (coloured points) and corresponding residuals (grey lines).

how kinetic models can be generated using structured data from similar automated flow systems, ${ }^{30,31}$ but kinetic models built from data obtained during a self-optimisation experiment have not been reported prior to this work. Ordinary differential equation (ODE) solvers were used to predict reaction progression under different conditions using a commonly accepted kinetic model for bi-molecular surface catalysed reactions, the Langmuir Hinshelwood (LH) model. ${ }^{32}$ This model is shown in (eqn (1)), where $S$ is nanoparticle surface area per unit volume, $k$ is the overall rate constant for the reaction, $C_{\mathrm{Nit}} / C_{\mathrm{BH}_{4}}$ represent the concentrations and $K_{\mathrm{Nit}} / K_{\mathrm{BH}_{4}}$ are the absorption coefficients of 4-NP and sodium borohydride respectively (ESI, $\dagger$ Section 6).

$$
v=\frac{\mathrm{d} C_{\mathrm{Nit}}}{\mathrm{d} t}=\frac{k S K_{\mathrm{Nit}} C_{\mathrm{Nit}} K_{\mathrm{BH}_{4}} C_{\mathrm{BH}_{4}}}{\left(1+K_{\mathrm{Nit}} C_{\mathrm{Nit}}+K_{\mathrm{BH}_{4}} C_{\mathrm{BH}_{4}}\right)^{2}}
$$

Kinetic models such as this can be used to predict the outcome of reactions under different conditions, even when alternative reactor configurations are used. ${ }^{33}$ A genetic algorithm ${ }^{34}$ was used to determine the kinetic parameters within the $\mathrm{LH}$ model by maximising the convergence of the predicted reaction outcomes to experimental data. Upon identifying this kinetic information, it was possible to use the model to predict reaction conversion under different experimental conditions. The model was used to predict the reaction kinetics of a AuNP catalysed 4-NP reduction reaction performed in a batch reactor with a two-fold increase in 4-NP concentration, compared to the flow optimisation study. The resulting extrapolated reaction kinetics showed good agreement with the model prediction, with a residual error of only $\pm 1.85 \%$, see Fig. 3 (ESI, $\dagger$ Section 6.1).

In summary, a continuous flow reactor platform has been developed for the efficient optimisation of nanoparticle catalysed reactions. Automated reactor controls and a self-optimising feedback loop were integrated into an automated flow reactor system. The effect of changing residence times, sodium borohydride concentrations and AuNP surface area were explored using the global optimisation algorithm SNOBFIT. The system was able to identify the optimum reaction conditions for maximum 4-NP conversion within a set operating window. Furthermore, the data gained during this optimisation process was used to obtain a kinetic model by employing ODE solvers and a genetic algorithm to fit kinetic parameters to the experimental data, thereby generating a response surface for the explored design space. The generated model was then validated by accurately predicting the outcome of a nanoparticle catalysed reduction reaction performed in batch. The system presented herein allows efficient determination of the optimal conditions for nanoparticle catalysed reactions. Integrating this system into automated flow reactors for nanoparticle synthesis, could also enable rapid determination of the conditions required for producing optimal catalytic nanoparticles as well as the best conditions for their respective catalysed reaction.

This work was supported by Zabeeda P. Aslam at the Leeds Electron Microscopy and Spectroscopy Centre (LEMAS). RAB was supported by the Royal Academy of Engineering under the Research Chairs and Senior Research Fellowships scheme (RCSRF1920\9\38). We also acknowledge support from the Henry Royce Institute (EPSRC grants: EP/P022464/1, EP/ R00661X/1), which funded the VXSF Facilities within the Bragg Centre for Materials Research at Leeds, the EPSRC via the Complex Particulates and Processes CDT program $\left(\mathrm{BH}, \mathrm{CP}^{3}\right.$ CDT), the Cognitive Chemical Manufacturing project (TWC, RAB, RL, EP/R032807/1) and the University of Leeds.

\section{Conflicts of interest}

There are no conflicts to declare.

\section{Notes and references}

1 Z.-Y. Zhou, N. Tian, J.-T. Li, I. Broadwell and S.-G. Sun, Chem. Soc. Rev., 2011, 40, 4167-4185.

2 Q. Wang and K. Domen, Chem. Rev., 2019, 120, 919-985.

3 T. Mitsudome and K. Kaneda, Green Chem., 2013, 15, 2636-2654.

4 T. Mallat and A. Baiker, Annu. Rev. Chem. Biomol. Eng., 2012, 3, 11-28.

5 A. Fihri, M. Bouhrara, B. Nekoueishahraki, J. M. Basset and V. Polshettiwar, Chem. Soc. Rev., 2011, 40, 5181-5203.

6 D. Astruc, Chem. Rev., 2020, 120, 461-463.

7 K.-J. Wu and L. Torrente-Murciano, React. Chem. Eng., 2018, 3, 267-276.

8 A. Monga and B. Pal, New J. Chem., 2015, 39, 304-313.

9 B. Roldan Cuenya, Thin Solid Films, 2010, 518, 3127-3150.

10 N. Narayan, A. Meiyazhagan and R. Vajtai, Materials, 2019, 12, 1-12. 11 A. D. Clayton, J. A. Manson, C. J. Taylor, T. W. Chamberlain, B. A. Taylor, G. Clemens and R. A. Bourne, React. Chem. Eng., 2019, 4, 1545-1554.

12 B. J. Reizman, Y.-M. Wang, S. L. Buchwald and K. F. Jensen, React. Chem. Eng., 2016, 1, 658-666.

13 L. M. Baumgartner, C. W. Coley, B. J. Reizman, K. W. Gao Ab and K. F. Jensen, React. Chem. Eng., 2018, 3, 301-311.

14 N. Holmes, G. R. Akien, A. J. Blacker, R. L. Woodward, R. E. Meadows and R. A. Bourne, React. Chem. Eng., 2016, 1, 366-371.

15 S. Krishnadasan, R. J. C. Brown, J. deMello and A. J. deMello, Lab Chip, 2007, 7, 1377-1612.

16 O. Długosz and M. Banach, React. Chem. Eng., 2020, 5, 1619-1641. 
17 E. J. Roberts, L. R. Karadaghi, L. Wang, N. Malmstadt and R. L. Brutchey, ACS Appl. Mater. Interfaces, 2019, 11, 27479-27502.

18 A. Sivo, R. d. S. Galaverna, G. R. Gomes, J. C. Pastre and G. Vilé, React. Chem. Eng., 2021, DOI: 10.1039/D0RE00411A.

19 V. Sebastian, S. Khan and A. Kulkarni, J. Flow Chem., 2017, 7, 96-105.

20 K.-J. Wu, G. M. De Varine Bohan and L. Torrente-Murciano, React. Chem. Eng., 2017, 2, 116-128.

21 H. Liu, J. Huang, D. Sun, O.-W. Tareque, J. Li and Q. Li, J. Nanopart. Res., 2014, 16, 1-9.

22 R. W. Epps, M. S. Bowen, A. A. Volk, K. Abdel-Latif, S. Han, K. G. Reyes, A. Amassian and M. Abolhasani, Adv. Mater., 2020, 32, 2001626.

23 E. Lester, P. Blood, J. Denyer, D. Giddings, B. Azzopardi and M. Poliakoff, J. Supercrit. Fluids, 2006, 37, 209-214.

24 J. S. Carey, D. Laffan, C. Thomson and M. T. Williams, Org. Biomol. Chem., 2006, 4, 2337-2347.

25 T. Aditya, A. Pal and T. Pal, Chem. Commun., 2015, 51, 9410-9431.
26 N. G. Bastus, J. Comenge and V. V. Puntes, Langmuir, 2011, 27, 11098-11105.

27 W. Huyer and A. Neumaier, ACM Trans. Math. Softw., 2008, 35, 20575-20587.

28 S. Wunder, F. Polzer, Y. Lu, Y. Mei and M. Ballauff, J. Phys. Chem. C, 2010, 114, 8814-8820.

29 P. Hervés, M. Pérez-Lorenzo, L. M. Liz-Marzán, J. Dzubiella, Y. Lu and M. Ballauff, Chem. Soc. Rev., 2012, 41, 5577-5587.

30 C. A. Hone, A. Boyd, A. O'Kearney-McMullan, R. A. Bourne and F. L. Muller, React. Chem. Eng., 2019, 4, 1565-1570.

31 C. J. Taylor, M. Booth, J. A. Manson, M. J. W. Willis, G. Clemens, B. A. Taylor, T. W. Chamberlain and R. A. Bourne, Chem. Eng. J., 2020, 127017.

32 W. H. Kang and H. Chuan Weinberg, Chem. Rev., 1995, 95, 667-676.

33 N. Zaborenko, M. W. Bedore, T. F. Jamison and K. F. Jensen, Org. Process Res. Dev., 2011, 15, 131-139.

34 D. E. Goldberg, Genetic Algorithms in Search, Optimization and Machine Learning, Addison-Wesley Longman Publishing Co., Inc., USA, 1st edn, 1989. 